\title{
Clinical Profile and Complications of Paracentesis in Refractory Ascites Patients With Cirrhosis
}

\author{
Sreenivasa Rao Sudulagunta ${ }^{\mathrm{a}, \mathrm{f}}$, Mahesh Babu Sodalagunta ${ }^{\mathrm{b}}$, Shiva Kumar Bangalore Raja ${ }^{\mathrm{c}}$, \\ Hadi Khorram ${ }^{\text {d }}$, Mona Sepehrare, Zahra Noroozpour ${ }^{\mathrm{a}}$
}

\begin{abstract}
Background: Large volume paracentesis is found to be safer and more effective for the treatment of tense ascites compared with largerthan-usual doses of diuretics according to studies. The objectives of the study was to evaluate patients with refractory ascites regarding clinical profile, technique of paracentesis, complications, amount of ascites drained, prognosis and co-morbid conditions associated with it.

Methods: Retrospective study was performed including patients between January 2011 and December 2013 with data pooled from total of five hospitals. A total of 4,389 paracenteses were performed on the 1,218 patients with a mean volume of $4,900 \pm 2,795 \mathrm{~mL}$ ascitic fluid drained. Blind technique, ultrasound-guided technique of paracentesis and pig tail catheter drainage were evaluated. Diabetes mellitus data from available patients and data regarding co-morbidities were analyzed. Coagulation abnormalities in patients were studied.
\end{abstract}

Results: Study group age ranged from 34 to 79 years, and alcohol is the main cause of cirrhosis. Dyslipidemia was observed in 1,080 patients $(88.66 \%)$. At the time of inclusion in the study, $40 \%$ of the patients had $\geq 2$ other cirrhosis-related complications and $20 \%$ of the study population had $\geq 3$ complications. Early complications occurred in $27.5 \%$ (337) of patients and late complications constituted $16.83 \%$ (205 patients).

Conclusions: Even with abnormal coagulation, paracentesis is a safe procedure. But significant co-morbidities should be addressed with care in cirrhosis patients. Ultrasound guidance during the procedure whenever required should be encouraged.

Manuscript accepted for publication June 10, 2015

${ }^{a}$ Dr.B.R.Ambedkar Medical College, Bangalore, India

${ }^{b} \mathrm{~K}$ S Hegde Medical College, India

'Department of Medicine, Dr.B.R.Ambedkar Medical College, Bangalore, India

dOtolaryngology Department, Dr.B.R.Ambedkar Medical College, Bangalore, India

eBaptist Hospital, Bangalore, India

${ }^{f}$ Corresponding Author: Sreenivas Rao Sudulagunta, Dr.B.R.Ambedkar Medical College, Shampura Main Road, Bangalore 560045, India.

Email: dr.sreenivas@live.in

doi: http://dx.doi.org/10.14740/gr661w
Keywords: Cirrhosis; Ascites; Paracentesis; Pig tail catheter; Ultrasound; Dyslipidemia

\section{Introduction}

Refractory ascites is defined as ascites that does not recede or that recurs shortly after therapeutic paracentesis, despite sodium restriction and diuretic treatment. Cirrhosis is defined histologically as a diffuse hepatic process characterized by fibrosis and the conversion of normal liver architecture into structurally abnormal nodules [1]. Cirrhosis can present with varied complications at one end of the spectrum that is asymptomatic, whereas on other end, complications such as hepatic encephalopathy, gastroesophageal varices and ascites. One of the danger signs of decompensated liver disease is refractory ascites, having a prevalence of $5-10 \%$ in patients with ascites [2] and survival rate as low as $50 \%$ at $6-12$ months postdiagnosis [3-5].

Refractory ascites refers to the inability to mobilize ascitic fluid with diagnostic criteria illustrated by Moore et al [6] (Table 1). Even when patients are on a sodium-restricted diet $(\leq 90 \mathrm{mmol} / \mathrm{day})$, excretion of sodium is less than what they consume [3]. Despite giving on maximum doses of diuretics (furosemide $160 \mathrm{mg}$ and spironolactone $400 \mathrm{mg}$ ) for at least 1 week, ascites persists with a mean weight loss of less than 0.8 $\mathrm{kg}$ over 4 days [3]. Multiple early recurrences of ascites, usually seen within 4 weeks of fluid mobilization, may be the first presentation of refractory ascites in many patients [3].

Table 1. Diagnostic Criteria of Refractory Ascites

1) Lack of response to maximal doses of diuretic for at least 1 week

2) Diuretic-induced complications in the absence of other precipitating factors

3) Early recurrence of ascites within 4 weeks of fluid mobilization

4) Persistent ascites despite sodium restriction

5) Mean weight loss $<0.8 \mathrm{~kg}$ over 4 days

6) Urinary sodium excretion less than sodium intake 
Table 2. Clinical Implications of Refractory Ascites

1) Dilutional hyponatremia

2) Hepatorenal syndrome

3) Spontaneous bacterial peritonitis

4) Hepatic hydrothorax

5) Spontaneous bacterial empyema

6) Umbilical hernia

Refractory ascites has a poor prognosis, and complications are illustrated in Table 2. Several large randomized controlled trials have shown that repeated large volume paracentesis (4 $6 \mathrm{~L}$ ) is safer procedure. It is considered more effective in tense ascites compared to larger-than-usual doses of diuretics [710]. Benefits observed were lower incidence of systemic and hemodynamic disturbance, electrolyte abnormalities, renal impairment and encephalopathy compared with diuretic therapy [7], also with improvement in cardiac output, lung volumes and reductions in intra-abdominal, portal intra-thoracic and pulmonary pressures.

\section{Aim of the study}

The aim of the study was as follows: 1) evaluate the complications arising from large volume paracentesis; 2) impact of the choice of needle type and usage of ultrasound in paracentesis; 3 ) amount of drained ascitic fluid and its role in complications; 4) prognosis of the patients who underwent the procedure.

\section{Materials and Methods}

Retrospective study was performed including patients between January 2011 and December 2013. These data were pooled from total of five hospitals with consent taken from all of them. Inclusion criteria were cirrhosis and presence of refractory ascites requiring therapeutic paracentesis. Refractory ascites was diagnosed by the Moore et al criteria illustrated in Table 1. Diagnosis of cirrhosis was based on clinical history, examination, ultrasound, CT abdomen, liver histology and laboratory data.

Exclusion criteria included patients with other co-morbid illnesses cancer, pancreatitis, heart failure, protein losing enteropathies and genetic disorders. A total of 875 patients were excluded due to unavailability of sufficient data of all investigations. The 1,218 patients were included in the study, and data were analyzed using information recorded in each procedure i.e., coagulation parameters prior to paracentesis, volume of drained ascitic fluid, ultrasound guidance, needle usage and type, pig tail catheter usage, immediate complications and dose of infused albumin.

The primary endpoint was occurrence of complications classified as: 1) early or immediate complications including ascitic fluid leak from the puncture site, local bleeding, technical problems (e.g., need for repuncture, slipping of the catheter from the abdominal wall, incomplete procedure) or complications requiring hospitalization within 7 days after the pro-
Table 3. Patient Characteristics

\begin{tabular}{|c|c|}
\hline Category & Number (\%) \\
\hline Age, median (range) & $56(34-79)$ \\
\hline \multicolumn{2}{|l|}{ Age category (years) } \\
\hline$<50$ & $204(17 \%)$ \\
\hline $50-59$ & $602(50.16 \%)$ \\
\hline $60-69$ & $214(17.83 \%)$ \\
\hline $70-79$ & $198(16.25 \%)$ \\
\hline$>80$ & 0 \\
\hline \multicolumn{2}{|l|}{ Gender } \\
\hline Male & $1,183(97.126 \%)$ \\
\hline Female & $35(2.873 \%)$ \\
\hline HbA1c, mean (available for 300 patients) & $8.3 \pm 2.09$ \\
\hline \multicolumn{2}{|l|}{ HbA1c category } \\
\hline$<6 \%$ & $29(9.66 \%)$ \\
\hline $6-6.9 \%$ & $60(20 \%)$ \\
\hline $7-7.9 \%$ & $120(40 \%)$ \\
\hline $8-8.9 \%$ & $69(23 \%)$ \\
\hline$>9 \%$ & $22(7.33 \%)$ \\
\hline Diabetes duration $>10$ years & $100(33.33 \%)$ \\
\hline Dyslipidemia & $1,080(88.66 \%)$ \\
\hline Abnormal LDL & $1,019(83.66 \%)$ \\
\hline Abnormal HDL & $721(59.19 \%)$ \\
\hline High total cholesterol & $1,001(82.18 \%)$ \\
\hline Triglycerides & $903(74.13 \%)$ \\
\hline Patients taking statins & $404(33.16 \%)$ \\
\hline
\end{tabular}

cedure; 2) other serious complications included hepatorenal syndrome (HRS), hepatic encephalopathy, spontaneous bacterial peritonitis (SBP) and large hematomas. The diagnosis of confirmed SBP requires an elevated ascitic fluid absolute polymorphonuclear leukocyte (PMN) count of at least 250 cells/ $\mathrm{mm}^{3}\left(0.25 \times 10^{9} / \mathrm{L}\right)$ and a positive ascitic fluid bacterial culture without an obvious intra-abdominal source of infection. SBP is considered if it occurred within 30 days of procedure.

Secondary endpoints were defined as: 1) the volume of ascitic fluid drained expressed as mean volume in $\mathrm{mL}$; and 2) patient's prognosis, which was defined as the patient's status on December 1, 2014. The cause of death was determined from the patient's record. P-values below 0.05 were considered significant, and 95\% confidence interval (CI) was used. Statistical analysis was done with $t$-test, Chi-square test and Fischer's exact test.

\section{Results}

Study group age ranged from 34 to 79 years and alcohol is the main cause of cirrhosis. Males are predominantly affected. Characteristics of patients were illustrated in Table 3. Males 
Table 4. Clinical and Laboratory Data

\begin{tabular}{ll}
\hline Age, years, median (range) & $56(34-79)$ \\
Sex & $\mathrm{n}(\%)$ \\
$\quad$ Male & $1,183(97.126 \%)$ \\
$\quad$ Female & $35(2.873 \%)$ \\
Etiology & $\mathrm{n}(\%)$ \\
$\quad$ Alcoholic & $1,151(94.49 \%)(\mathrm{F}: 28 ; \mathrm{M}: 1,123)$ \\
$\quad$ Hepatitis C-related & $16(1.313 \%)(\mathrm{F}: 2 ; \mathrm{M}: 14)$ \\
$\quad$ Hepatitis B-related & $42(3.448 \%)(\mathrm{F}: 3 ; \mathrm{M}: 39)$ \\
$\quad$ Cryptogenic & $9(0.738 \%)(\mathrm{F}: 2 ; \mathrm{M}: 7)$ \\
Serum albumin, g/dL & $26(14.3-38.0)$ \\
Serum bilirubin, $\mu$ mol/L, median (range) & $31(3.0-304.0)$ \\
Serum creatinine, $\mu$ mol/L, median (range) & $87(37-379)$ \\
INR, median (range) & $1.5(1.0-3.0)$ \\
Child-Pugh score, median (range) & $10(8-15)$ \\
Model for end-stage liver disease & $14.5(6.2-28.9)$ \\
(MELD) score, median (range) & \\
\hline
\end{tabular}

constitute $97 \%$ of the study group and females about $3 \%$. The $87 \%$ of the study population is above the age of 50 years. In patients with diabetes mellitus 300 patients' data were available. Poor control of diabetes was observed with about $70 \%$ of patients with glycosylated hemoglobin (HBA1C) more than $7 \%$, and HBA1C mean was $8.3 \pm 2.09$. The 100 patients $(33.33 \%)$ out of 300 patients with diabetes had diabetes for more than 10 years.

Dyslipidemia was observed in 1,080 patients $(88.66 \%)$ which is statistically significant than normal population $(\mathrm{P}<$ 0.001). In patients with dyslipidemia, abnormal low density lipoprotein (LDL) was found in 1,019 patients (83\%), abnormal high density lipoprotein (HDL) was found in 721 patients $(59 \%)$ and hypertriglyceridemia in 903 patients (74\%). Patients taking statins constituted only 33\% of patients (404), which is statistically significant $(\mathrm{P}<0.001)$ and alarming.

Alcoholism constituted $94.5 \%$ (1,151 patients) of the cause of cirrhosis of liver and ascites. Hepatitis B was associated with 42 patients $(3.44 \%)$, hepatitis $\mathrm{C}$ with 16 patients $(1.31 \%)$ and cryptogenic cirrhosis in $0.73 \%$ (nine) of patients. Majority of the patients included in the study had cirrhosis in advanced stage with a high Child-Pugh score median score of 10 (range 8 - 15) and mean $10.5,95 \%$ CI. The $73 \%$ belong to Child-Pugh class C. Clinical and laboratory data are illustrated

Table 5. Co-morbid Conditions in Patients

\begin{tabular}{ll}
\hline Co-morbidities & Number (\%) \\
\hline CKD & $153(12.56 \%)$ \\
\hline IHD & $209(16.50 \%)$ \\
Cerebrovascular disease & $104(8.53 \%)$ \\
COPD & $99(8.12 \%)$ \\
$\geq 2$ co-morbidities & $287(23.56 \%)$ \\
\hline
\end{tabular}

\section{in Table 4.}

At the time of inclusion in the study, $40 \%$ of the patients had $\geq 2$ other cirrhosis-related complications, and $20 \%$ of the study population had $\geq 3$ complications including esophageal varices, hepatic encephalopathy, hepatocellular carcinoma, hepatorenal syndrome, hepatopulmonary syndrome and spontaneous bacterial peritonitis. Co-morbid conditions were associated with $>50 \%$ of patients in study group (Table 5 ). The $16.5 \%$ patients (209) had ischemic heart disease (IHD), $12.5 \%$ (153) had chronic kidney disease (CKD), 8.5\% (104) patients had cerebrovascular disease and 8\% (99) had chronic obstructive pulmonary disease. Patients with $\geq 2$ co-morbidities constituted $23.5 \%$ (287 patients) of the study group.

A total of 4,389 paracenteses were performed on the 1,218 patients with a mean volume of 4,900 $\pm 2,795 \mathrm{~mL}$ ascitic fluid drained. Early complications occurred in $27.5 \%$ (337) of patients which are illustrated in Table 6. Early complications observed were incomplete drainage, pain at puncture site, local bleeding, hypotension, increased shortness of breath (SOB), re-punctures done, slipping of catheter used for tapping and leakage of ascitic fluid from puncture site. Late complications constituted $16.83 \%$ (205 patients). Late complications observed were fever, abdominal hematoma, hepatic encephalopathy, spontaneous bacterial peritonitis, hepatorenal syndrome and hepatopulmonary syndrome (Table 6). Out of these complications $7 \%$ were due to technical errors during performance or after the procedure.

Intravenous catheter was used in 4,189 paracenteses with $4,089(93.16 \%)$ being blind procedures where the dependent portion of ascites was drained (Table 7) [11]. The $100(2.27 \%)$ paracenteses were performed using ultrasound guidance. The complication rate in ultrasound-guided procedure was $0 \%$ compared to blind procedure which is statistically significant $(\mathrm{P}<0.001)$. In all these ultrasound-guided procedures paracentesis needle was used. In $200(4.55 \%)$ procedures pig tail cath- 
Table 6. Variables and Paracenteses Percentages

\begin{tabular}{|ll|}
\hline Calculated variables & Paracenteses $(\mathbf{n}=\mathbf{4 , 3 8 9})$ \\
\hline Early complications & $\mathrm{n}(\%)$ \\
\hline Incomplete drainage & $69(5.66 \%)$ \\
\hline Pain at puncture site & $57(4.67 \%)$ \\
\hline Local bleeding & $35(2.87 \%)$ \\
\hline Hypotension & $51(4.18 \%)$ \\
\hline Increased SOB & $30(2.46 \%)$ \\
\hline Re-punctures done & $32(2.62 \%)$ \\
\hline Slipping of catheter used for tapping & $24(1.97 \%)$ \\
\hline Leakage of ascitic fluid from puncture site & $39(3.20 \%)$ \\
Total & $337(27.66 \%)$ \\
\hline Late complications & $\mathrm{n}(\%)$ \\
\hline Fever & $23(1.88 \%)$ \\
Abdominal hematoma & $30(2.46 \%)$ \\
\hline Hepatic encephalopathy & $88(7.22 \%)$ \\
Spontaneous bacterial peritonitis & $32(2.62 \%)$ \\
Hepatorenal syndrome & $17(1.39 \%)$ \\
\hline Hepatopulmonary syndrome & $15(1.23 \%)$ \\
Mean volume \pm SD of drained ascitic fluid, mL & $4,900 \pm 2,795 \mathrm{~mL}$ \\
Total & $205(16.83 \%)$ \\
\hline
\end{tabular}

eter was used with higher rate of complications compared to intravenous catheter usage procedures, although it was not statistically significant. Early complications with pig tail catheter were $37(18.5 \%)$ compared to intravenous catheter procedure with $300(7.33 \%)$. Late complications with pig tail catheter were $8 \%$ compared to $5 \%$ of intravenous catheter procedure.

\section{Discussion}

Paracentesis is a word derived from Greek $\kappa \varepsilon v \tau \alpha ́ \omega$ ("to pierce"), a common procedure done in outpatient department and medical wards. According to recent studies mild hematological abnormalities do not increase risk of bleeding significantly $[12,13]$. Risk of bleeding increases with the following
[14]: 1) prothrombin time $>21 \mathrm{~s} ; 2$ ) international normalized ratio $\left(\right.$ INR) $>1.6$ and 3 ) platelet count $<50,000 / \mathrm{mm}^{3}$. Acute abdomen requiring surgery is an absolute contraindication. Relative contraindications are pregnancy, distended urinary bladder, abdominal wall cellulitis, distended bowel and intraabdominal adhesions [15].

Intravenous albumin infusions have been shown in recent studies to reduce the rate of complications by preventing postparacentesis-induced circulatory dysfunction (PICD). In spite of the benefits, still procedure is associated with many complications [16]. Complications found in this study were similar to other retrospective and prospective studies, even though few complications were minimal such as bleeding, slippage of catheter were less common $[17,18]$.

Late and serious complications associated with paracente-

Table 7. Ascitic Tap Procedure Followed in the Study [11]

Explain the procedure to the patient, including risks, and obtain consent

Position the patient, usually in the supine position with the head of the bed elevated to allow fluid to accumulate in the patient's lower abdomen

Position of the tap

Locate area of flank dullness lateral to the rectus abdominis muscle and go approximately $5 \mathrm{~cm}$ superior and medial to the anterior superior iliac spines

Avoid the inferior epigastric vessels which run up the side of the rectus abdominis to anastomose with the superior epigastric vessels coming down

Avoid the pelvic area, solid tumor masses, prominent superficial veins (caput medusa) and scars (may have collateral vessels close by or adherent bowel beneath) 
sis were hepatorenal syndrome, hepatopulmonary syndrome, spontaneous bacterial peritonitis and hepatic encephalopathy. These complications were observed but uncommonly in the study.

Higher incidence of complications with pig tail catheter compared to intravenous catheter drainage of ascitic fluid was observed. This observation is reinforced by other studies in 2001 by Schlottmann et al and Shaheen and Grimm, which found a significantly lower rate of re-punctures $(1: 6, \mathrm{P}=0.046)$ when using a special paracentesis needle [19, 20]. But the mean ascitic fluid was $250 \mathrm{~mL}$ more drained through pig tail catheter compared to intravenous catheter needle. The high incidence of complications in the pig tail catheter group may be due to excess and rapid drainage.

We found that ultrasound-guided ascitic tap is safer than blind procedure and using pig tail catheter. Using paracentesis needle is also accounted for the benefit in the patients who have undergone ultrasound-guided ascitic fluid drainage. U1trasound-guided paracentesis is particularly useful with minimal-to-moderate ascites, access is difficult and complications were expected due to drainage of the ascitic fluid described by Gottardi et al [21]. Few cases are reported regarding deaths due to intra-peritoneal hemorrhage due to paracentesis even under ultrasound guidance.

Our study found these significant observations. 1) Paracentesis is a relatively safe procedure with low complication rate. 2) Ultrasound-guided paracentesis was observed to have low complication rate even though blind paracentesis procedure is relatively safe with less complications. 3) No deaths were observed during and up to 1 month of paracentesis procedure attributable to the procedure. 4) Even though around $50 \%$ of patients had aberrant coagulation parameters, the complications of bleeding were significantly low. 5) As it is well known, alcohol is the major cause of cirrhosis of liver. 6) Poor control of diabetes was observed among the cirrhotic patients undergoing paracentesis. 7) Co-morbidities like cerebrovascular disease, IHD, and CKD are significantly higher in cirrhotic patients. 8) Almost more than $90 \%$ patients were dyslipidemic with low statins usage which should be addressed as the mortality increases.

\section{Conclusions}

Even with abnormal coagulation, paracentesis is a safe procedure. But significant co-morbidities should be addressed with care in cirrhosis patients. Ultrasound guidance during the procedure whenever required should be encouraged.

\section{Grant Support}

None.

\section{Conflict of Interest}

None.

\section{References}

1. Arroyo V. Pathophysiology, diagnosis and treatment of ascites in cirrhosis. Ann Hepatol. 2002;1(2):72-79.

2. Ring-Larsen H. Therapy of Ascites and Renal Dysfunction in Cirrhosis. Malden, Massachusetts: Blackwell Co; 1999. Treatment of refractory ascites in cirrhosis; pp. 480-491.

3. Cardenas A, Arroyo V. Refractory ascites. Dig Dis. 2005;23(1):30-38.

4. Moreau R, Delegue P, Pessione F, Hillaire S, Durand F, Lebrec D, Valla DC. Clinical characteristics and outcome of patients with cirrhosis and refractory ascites. Liver Int. 2004;24(5):457-464.

5. Guardiola J, Baliellas C, Xiol X, Fernandez Esparrach G, Gines P, Ventura P, Vazquez S. External validation of a prognostic model for predicting survival of cirrhotic patients with refractory ascites. Am J Gastroenterol. 2002;97(9):2374-2378.

6. Moore KP, Wong F, Gines P, Bernardi M, Ochs A, Salerno $\mathrm{F}$, Angeli $\mathrm{P}$, et al. The management of ascites in cirrhosis: report on the consensus conference of the International Ascites Club. Hepatology. 2003;38(1):258-266.

7. Gines P, Arroyo V, Quintero E, Planas R, Bory F, Cabrera $\mathrm{J}$, Rimola A, et al. Comparison of paracentesis and diuretics in the treatment of cirrhotics with tense ascites. Results of a randomized study. Gastroenterology. 1987;93(2):234241.

8. Salerno F, Badalamenti S, Incerti P, Tempini S, Restelli B, Bruno S, Bellati G, et al. Repeated paracentesis and i.v. albumin infusion to treat 'tense' ascites in cirrhotic patients. A safe alternative therapy. J Hepatol. 1987;5(1):102-108.

9. Pinto PC, Amerian J, Reynolds TB. Large-volume paracentesis in nonedematous patients with tense ascites: its effect on intravascular volume. Hepatology. 1988;8(2):207210.

10. Sola R, Vila MC, Andreu M, Oliver MI, Coll S, Gana J, Ledesma S, et al. Total paracentesis with dextran 40 vs diuretics in the treatment of ascites in cirrhosis: a randomized controlled study. J Hepatol. 1994;20(2):282-288.

11. Wong CL, Holroyd-Leduc J, Thorpe KE, Straus SE. Does this patient have bacterial peritonitis or portal hypertension? How do I perform a paracentesis and analyze the results? JAMA. 2008;299(10):1166-1178.

12. McVay PA, Toy PT. Lack of increased bleeding after paracentesis and thoracentesis in patients with mild coagulation abnormalities. Transfusion. 1991;31(2):164-171.

13. Moore KP, Aithal GP. Guidelines on the management of ascites in cirrhosis. Gut. 2006;55(Suppl 6):vi1-12.

14. Gines P, Cardenas A, Arroyo V, Rodes J. Management of cirrhosis and ascites. N Engl J Med. 2004;350(16):16461654.

15. Paracentesis, Author: Gil Z Shlamovitz, MD; Chief Editor: Rick Kulkarni, MD. http://emedicine.medscape.com/ article/80944-overview\#showall.

16. Gines P, Tito L, Arroyo V, Planas R, Panes J, Viver J, Torres $\mathrm{M}$, et al. Randomized comparative study of therapeutic paracentesis with and without intravenous albumin in cirrhosis. Gastroenterology. 1988;94(6):1493-1502. 
17. Runyon BA. Paracentesis of ascitic fluid. A safe procedure. Arch Intern Med. 1986;146(11):2259-2261.

18. Webster ST, Brown KL, Lucey MR, Nostrant TT. Hemorrhagic complications of large volume abdominal paracentesis. Am J Gastroenterol. 1996;91(2):366-368.

19. Schlottmann K, Gelbmann C, Grune S, Lock G, Scholmerich J. [A new paracentesis needle for ascites and pleural effusion compared with the venous indwelling catheter. A prospective, randomized study]. Med Klin (Munich).
2001;96(6):321-324.

20. Shaheen NJ, Grimm IS. Comparison of the Caldwell needle/cannula with Angiocath needle in large volume paracentesis. Am J Gastroenterol. 1996;91(9):1731-1733.

21. De Gottardi A, Thevenot T, Spahr L, Morard I, BressonHadni S, Torres F, Giostra E, et al. Risk of complications after abdominal paracentesis in cirrhotic patients: a prospective study. Clin Gastroenterol Hepatol. 2009;7(8):906909. 